(C) [2008] IEEE. Reprinted, with permission, from [Hoang T. Trieu, Hung T. Nguyen and Keith Willey, Advanced Obstacle Avoidance for a Laser Based Wheelchair Using Optimised Bayesian Neural Networks, Engineering in Medicine and Biology Society, 2008. EMBS 2008. 30th Annual International Conference of the IEEE, 20-25 Aug. 2008]. This material is posted here with permission of the IEEE. Such ermission of the IEEE does not in any way imply IEEE endorsement of any of the University of Technology, Sydney's products or services. Internal or personal use of this material is permitted. However, permission to reprint/republish this material for advertising or promotional purposes or for creating new collective works for resale or redistribution must be obtained from the IEEE by writing to pubs-permissions@ieee.org. By choosing to view this document, you agree to all provisions of the copyright laws protecting it 


\title{
Advanced Obstacle Avoidance for a Laser Based Wheelchair Using Optimised Bayesian Neural Networks
}

\author{
Hoang T. Trieu, Hung T. Nguyen, Senior Member, IEEE and Keith Willey, Member, IEEE
}

\begin{abstract}
In this paper we present an advanced method of obstacle avoidance for a laser based intelligent wheelchair using optimized Bayesian neural networks. Three neural networks are designed for three separate sub-tasks: passing through a door way, corridor and wall following and general obstacle avoidance. The accurate usable accessible space is determined by including the actual wheelchair dimensions in a real-time map used as inputs to each networks. Data acquisitions are performed separately to collect the patterns required for specified sub-tasks. Bayesian frame work is used to determine the optimal neural network structure in each case. Then these networks are trained under the supervision of Bayesian rule. Experiment results showed that compare to the VFH algorithm our neural networks navigated a smoother path following a near optimum trajectory.
\end{abstract}

\section{INTRODUCTION}

$\mathrm{R}$ eports show that the number of elderly people and people with disabilities are increasing significantly [1]. The aim of Rehabilitation technology is to improve the quality of life for people with disabilities. In particular, intelligent wheelchairs are developed to accommodate people with mobility impairments. The provision of independent mobility, also increases the opportunities for users to develop physical, cognitive, communication and social skills [2].

Obstacle avoidance is one of the most fundamental tasks of autonomous systems. It is also a very important function in designing an intelligent wheelchair system with its specific requirements such as safety, smoothness, and comfort. Many algorithms for enabling autonomous tasks have been developed. The most popular being, global map, occupancy grid [3], virtual force field and vector field histogram $[4,5]$. However, most of these algorithms have difficulty successfully operating in dense and dynamic environments and providing smooth trajectories and stability required by any assistive wheelchair systems. Our Bayesian learning general obstacle avoidance neural network was first introduced in [6]. Initial results were encouraging but also showed that a single neural network could not provide the

This work was supported in part by Australian Research Council under Discovery Grant DP0666942 and LIEF Grant LE0668541.

Hoang T. Trieu is with Faculty of Engineering, University of Technology, Sydney, Broadway, NSW 2007, Australia (phone: +612-95142451; fax: +61 29514 2868; e-mail: thtrieu@eng.uts.edu.au).

Hung T. Nguyen is with Faculty of Engineering, University of Technology, Sydney, Broadway, NSW 2007, Australia (e-mail: Hung.Nguyen@uts.edu.au).

Keith Willey is with Faculty of Engineering, University of Technology, Sydney, Broadway, NSW 2007, Australia (e-mail: kwilley@eng.uts.edu.au). desired performance in all situations and hence further development was required.

In this paper, we present a more advanced obstacle avoidance technique that utilises separate neural networks for specified tasks. The obstacle avoidance task is divided into three sub-tasks: passing through a door, corridor and wall following and general obstacle avoidance. This enables the network to respond to the particular features of each task, improving performance. Specific data acquisitions are performed to collect the patterns used to design the neural network for each task. Bayesian framework is then applied to determine the optimal network structures. The training patterns are then used in conjunction with the Bayesian training process to improve the generalisation and performances of each network. Our method was able to successfully accomplish difficult navigation tasks smoothly following a near optimum trajectory.

This paper will be presented in a number of sections. The next section reviews the Bayesian framework. The following two sections discuss the obstacle avoidance method and present the results. In the last section we present our discussion and conclusions.

\section{BAYESIAN NEURAL NETWORK}

\section{A. Regularisation}

In network training, using simple sum square of error as a performance function can not prevent the over-growth of network's weights. Large weight values can produce poor performance with new data. An appropriate regulation strategy can be achieved by adding a weight decay term to the error function, $E_{D}$, to penalise the growth of weights as follow:

$$
S(w)=\beta E_{D}+\sum_{i=1}^{G} \alpha_{i} E_{w_{i}},
$$

where $S(w)$ is the performance function, $\beta$ and $\alpha_{i}$ are known as the hyper-parameters for training and weight sets, $E_{W i}$ is the weight values for the $i^{\text {th }}$ group of weights and biases, and $G$ is the number of groups of weights and biases in the neural network. Minimising this performance function, $S(w)$, by appropriately adjusting the hyper-parameters can converge the weight set to a global minimum ensuring both a network's performance and generalisation.

\section{B. Bayesian Framework}

The Bayesian framework for a multi-layer perceptron neural network is based on a Gaussian approximation, [7, 8]. It automatically adjusts the hyper-parameters to the most probable value given by the training data set during the 
Bayesian learning process. Different networks with different structures and trained weight sets can be compared and ranked to find the most suitable network for an application.

According to the Bayesian inference, the posterior probability of the network parameters, weight set $-w$, of a neural network, $H$, given by a training data set, $D$, could be estimated by:

$$
p(w \mid D)=\iint p(w \mid \alpha, \beta, D) p(\alpha, \beta \mid D) d \alpha d \beta .
$$

With a Gaussian approximation for posterior distribution of hyper-parameters, $p(\alpha, \beta \mid D)$, this integration can estimated as

$$
p(w \mid D) \propto p\left(w, \alpha_{M P}, \beta_{M P}, D\right) \iint p(\alpha, \beta \mid D) d \alpha d \beta
$$

which can be simplified to $p\left(w, \alpha_{M P}, \beta_{M P}, D\right)$ by using $\iint p(\alpha, \beta \mid D) d \alpha d \beta=1$ as a normalization factor [7].

This mean that the most probable values $\alpha_{M P}, \beta_{M P}$ shall maximise the posterior probability of weights. These values, $\alpha_{M P}, \quad \beta_{M P}$, can be estimated from their posterior of distribution as equation follows, [9].

$$
p(\alpha, \beta \mid D)=\frac{p(D \mid \alpha, \beta) p(\alpha, \beta)}{p(D)} \propto p(D \mid \alpha, \beta)
$$

The term $p(D \mid \alpha, \beta)$ is called evidence of the hyperparameters. The log of this evidence could be estimated by equation bellows, [9]:

$$
\ln p(D \mid \alpha, \beta)=-S\left(w^{M P}\right)-\frac{1}{2} \ln |A|+\frac{W}{2} \ln \alpha+\frac{N}{2} \ln \beta-\frac{N}{2} \ln (2 \pi)
$$

where $A$ is the Hessian matrix of the cost function, $A=\alpha C+$ $\beta B, \nabla \nabla E_{w}=C, \nabla \nabla E_{D}=B$. The term $W$ is the number of network parameters, $N$ is the number of training patterns and $w^{M P}$ is the most probable value of weight.

The most probable values of hyper-parameters $\alpha^{M P}, \beta^{M P}$ can be estimated by equation above as:

$$
\alpha^{M P}=\frac{\gamma}{2 E_{W}^{M P}}, \beta^{M P}=\frac{N-\gamma}{2 E_{D}^{M P}}, \gamma=\sum_{i=1}^{W} \frac{\lambda_{i}}{\lambda_{i}+\alpha}
$$

where $\lambda_{i}$ is the eigenvalue of the Hessian matrix $A$. These values are re-estimated during training to constrain the over growth of weight values to ensure the generalization of the neural network.

Bayesian framework can compare and rank different neural networks with different structures and weight values by estimating the probabilities of these networks. The Bayesian formula for a network, $H_{i}$, and its probability given by the training data, $D$, is

$$
p\left(H_{i} \mid D\right)=\frac{p\left(D \mid H_{i}\right) p\left(H_{i}\right)}{p(D)} \propto p\left(D \mid H_{i}\right) .
$$

The prior probability of a network is assumed to be the same for all models and the term $p(D)$ is independent on the model. Hence, the posterior probability of the model can be determined by evidence $p\left(D \mid H_{i}\right)$. The evidence of the model can be calculated by estimating the integration below over the set of network parameters $-w$,

$$
p\left(D \mid H_{i}\right)=\int p\left(D \mid w, H_{i}\right) p\left(w, H_{i}\right) d w .
$$

Bishop evaluated the log evidence of model, $H_{i}$, rather than the evidence itself [9] as:

$$
\begin{gathered}
\ln p\left(D \mid H_{i}\right)=-\alpha_{M P} E_{W}^{M P}-\beta_{M P} E_{D}^{M P}-\frac{1}{2} \ln |A|+\frac{W}{2} \ln \alpha_{M P}+ \\
+\frac{N}{2} \ln \beta_{M P}+\ln M !+2 \ln M+\frac{1}{2} \ln \frac{2}{\gamma}+\frac{1}{2} \ln \frac{2}{N-\gamma}(8)
\end{gathered}
$$

The different network structures are compared by estimating the evidence by the above equation. The optimal network is the one that has the highest evidence.

\section{OBSTACLE AVOIDANCE METHOD}

\section{A. Data Acquisition}

Our neural networks use usable accessible space data as an input and providing values of steering and velocity as outputs. A method reported in [10] for accurately determining collision free accessible space, by combining information from a laser range finder, the actual wheelchair dimensions and encoder sensors mounted on the wheelchair is used to produce a real-time map as shown in fig. 1 .

The wheelchair is required to follow a number of predetermined paths to gather data for training. These paths are selected by the designer to simulate the previously mentioned tasks. The movements of the wheelchair are measured and formed as training patterns for each obstacle avoidance sub-task.

\section{B. Bayesian Training}

The Bayesian framework is first applied to determine the most suitable structure of a neural network for each task by estimating the evidence of a set of neural networks with different hidden nodes by equation 8 . The collected patterns are divided to two sets: training and testing sets. The aim of using a testing set is to verify generalisation of these networks. Second, all available patterns are used in training this network under the Bayesian rule to find the most probable weight set that improves the network's performances and generalization. The trained networks are then used to control the wheelchair in real-time.

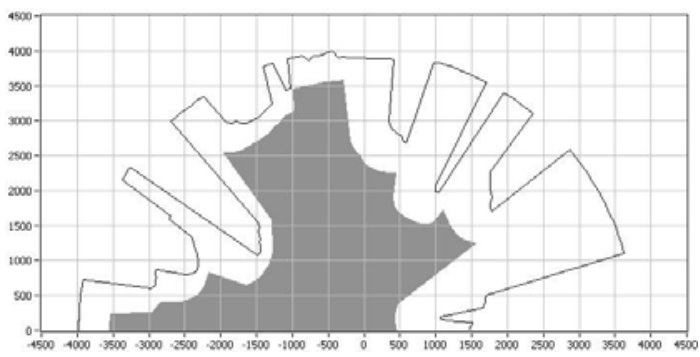

Fig. 1. Useable accessible space identified as the shaded area and real-time obstacle map (outer line) for the wheelchair.

\section{EXPERIMENT RESULTS}

\section{A. General Obstacle Avoidance (GOA)}

The wheelchair was made to follow a number of paths classified as general obstacle avoidance situations to collect 
data. The number of patterns gathered was 3951. This set was divided to two sets: training and test (2374 and 1577 patterns respectively) based on the independent data collected from the different paths.

First, a Bayesian framework was applied to determine the most suitable structure for the general obstacle avoidance neural network. The training results are shown in fig. 2 . The network with four hidden nodes produced the highest evidence. This network also produced lower errors compared to other networks when tested by the test set. Hence this structure is the most suitable for the general obstacle avoidance task.

Second, the data from both the training and test sets was used to train a network with four hidden nodes applying the Bayesian rule. During training, the Bayesian framework constrains the growth of weights to the most probable values by automatically adjusting the hyper-parameters, $\alpha$ and $\beta$. After training the network was used enable the wheelchair to perform general obstacle avoidance tasks.

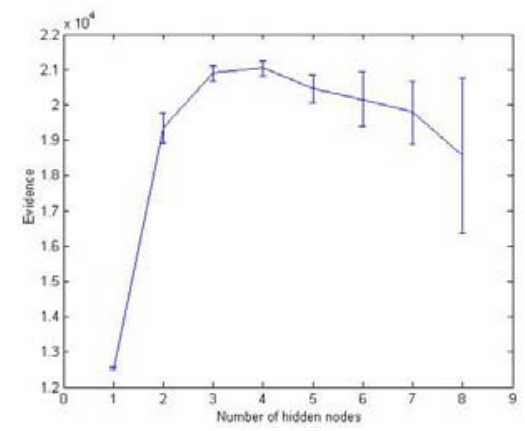

Fig. 2. GOA task's training result. The network with four hidden nodes is the most suitable also providing low testing errors.

In the first experiment (results shown in fig. 3) the wheelchair was asked to access to a narrow dead-end corridor. Our method was compared to the well-known VFH [5], Vector Field Histogram. The VFH algorithm utilises a polar-histogram of range data to keep to the middle of the available free-space determined by a constant threshold. As shown in the figure our neural network method produced a superior result providing a smooth, stable and reliable trajectory as the wheelchair navigated the requested path. Conversely, the VHF algorithm was not as smooth and guided the wheelchair extremely close to the obstacle on the left hand side when negotiating the corner.

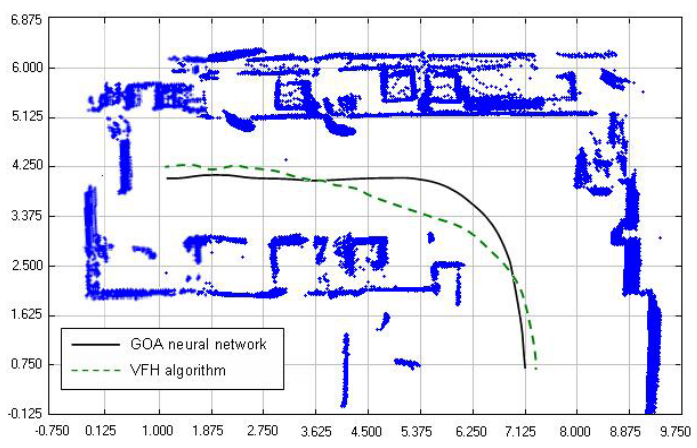

Fig. 3. GOA neural network' performances.

\section{B. Corridor and Wall Following (C-WF)}

The data acquisition procedure as described for the general obstacle avoidance task was again used for this task. The number of collected patterns was 3478. These were divided into separate training and test sets (2283 and 1195 patterns for each set respectively).

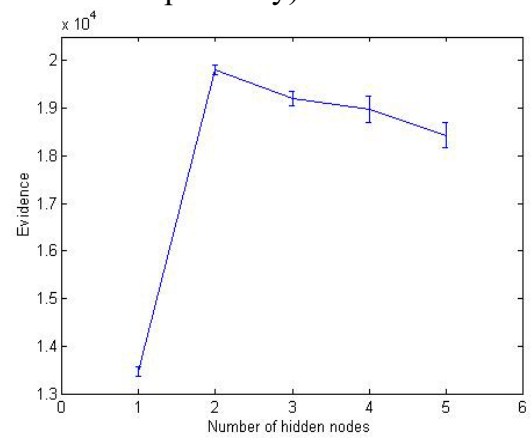

Fig. 4. C-WF task's training result. The network with only two hidden nodes can provide the highest evidence and low test errors.

The Bayesian framework was again applied to find the most suitable network structure for this task. The training results shown in fig. 4 found that a network with only two hidden nodes produced the highest evidence and recorded consistently low errors when tested by the test set. Hence this is the most suitable network structure for the corridor/ wall following task. The most probable weight values were obtained by applying all patterns to the Bayesian training. The trained network then was used to control the wheelchair when performing corridor and wall following tasks.

The wheelchair was required to travel along the longest wall in our laboratory as shown in fig. 5. Again the performance of our neural network method was compared to the VFH algorithm. Our method guided the wheelchair smoothly and reasonably directly along the wall, moving only slightly away from the wall where the wider free-space was encountered. Conversely, the VFH algorithm produced a less satisfactory result producing a fluctuating and less direct path during the experiment.

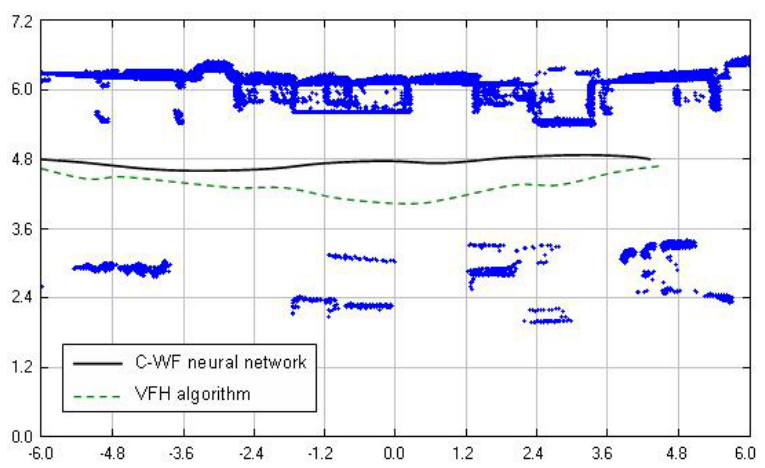

Fig. 5. The corridor and wall following experiment showed our neural network provided superior performance compared to VFH algorithm.

\section{Door Passing (DP)}

Of the three sub-tasks passing through a doorway is the most difficult. 3070 patterns were acquired during the day to 
requisition process, which were divided into training and test sub-set with 2356 and 714 patterns respectively.

After applying Bayesian training, a network with five hidden nodes was found to produce the highest evidence, fig. 6 and small errors when tested by the test set. The most probable weight values were obtained by applying all patterns to the Bayesian training of the five hidden node network. After training this neural network was used to control the wheelchair for door passing tasks.

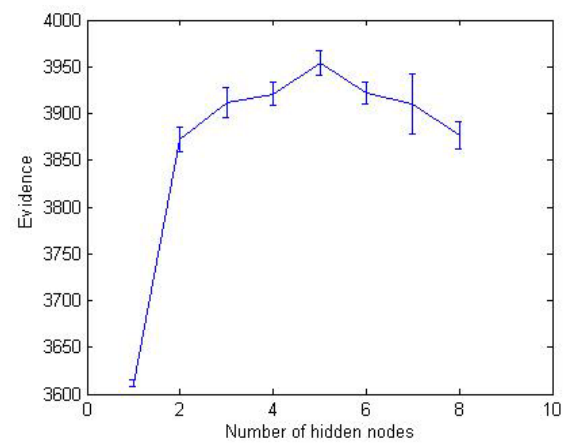

Fig. 6. Passing through a door task's training result. The network with five hidden nodes produced the highest evidence and provided low error when tested by the testing set.

To test the network performance the wheelchair was requested to travel along a corridor and then turn through a doorway, fig. 7. The experiment found our network had superior performance compared to the VFH algorithm. This network made a substantial turn as the wheelchair approached the doorway then reduced the wheelchair's velocity at point 1 in preparation for the door passing task. The wheelchair followed a smooth and near optimal path in negotiating the doorway finishing the task at point 2 , after which the wheelchair moved towards the middle of the available free-space. Conversely, the VFH algorithm was unable to successfully navigate through the doorway, colliding with the door frame before stopping at point 3 .

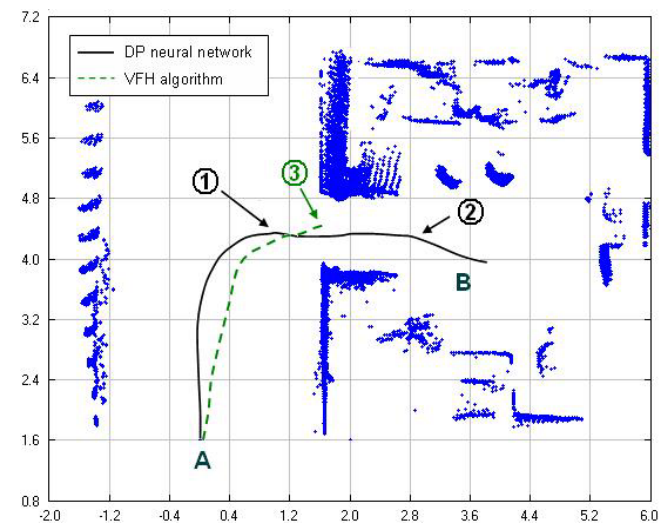

Fig. 7. Our network allowed the wheelchair to navigate safely and relatively directly through the doorway. In comparison the VHF algorithm crashed the wheelchair into the door frame.

\section{DISCUSSION AND CONCLUSION}

The results suggest that Bayesian neural networks have significant potential to solve the obstacle avoidance tasks required by an intelligent wheelchair system. Improved performance is achieved by dividing the overall obstacle avoidance task into a number of sub-tasks each controlled by using the specifically designed neural networks. In addition, as the Bayesian framework resists overgrowth of network weights, it promotes network generalization, assisting it to deal with new environments. After training the networks showed the potential to provide satisfactory realtime performance. The optimal method of effectively combining these networks to achieve the desired performance is the focus of ongoing research.

We are currently working on developing a shared control strategy to combine user intentions via an interface, such as a joystick or head movement sensor with available free space and safety considerations to accurately interpret user's intensions. These intensions will be used to decide which of the available sub-tasks should be chosen in any given situation, allowing the wheelchair to successfully navigate in dense and dynamic environments. We are currently investigating the potential of using a Bayesian framework [11] to develop an effective strategy based on probabilistic reasoning.

\section{REFERENCES}

[1] S. P. Parikh, V. Grassi Jr., V. Kumar, and J. Okamoto Jr., "Integrating Human Inputs with Autonomous Behaviors on an Intelligent Wheelchair Platform," IEEE Intelligent Systems, vol. 22, pp. 33 - 41, 2007.

[2] R. C. Simpson, "Smart wheelchairs: A literature review," Journal of Rehabilitation Reasearch \& Development, vol. 42, pp. 423-436, 2005.

[3] A. Elfes, "Using occupancy grids for mobile robot perception and navigation," Computer, vol. 22, pp. 46 - 57, 1989.

[4] J. Borenstein and Y. Koren, "Real-time obstacle avoidance for fast mobile robots in cluttered environments," IEEE International Conference on Robotics and Automation, vol. 1, pp. 572 - 577, 1990.

[5] J. Borenstein and Y. Koren, "The vector field histogram-fast obstacle avoidance for mobile robots," IEEE Transactions on Robotics and Automation, vol. 7, pp. 278 - 288, 1991.

[6] H. T. Trieu, H. T. Nguyen, and K. Willey, "Obstacle Avoidance for Power Wheelchair Using Bayesian Neural Network," 29th Annual International Conference of the IEEE Engineering in Medicine and Biology Society, pp. 4771 - 4774, 2007.

[7] D. J. C. MacKay, "Bayesian interpolation," Neural Computation, vol. 4, pp. 415-447, 1992a.

[8] D. J. C. MacKay, "Bayesian neural networks and density networks," Nuclear Instruments and Methods in Physics Research, pp. 73-80, 1995.

[9] C. M. Bishop, Neural networks for pattern recognition. Oxford: Oxford University Press, 1995.

[10] H. T. Trieu, H. T. Nguyen, and K. Willey, "Shared Control Strategies for Obstacle Avoidance Tasks in an Intelligent Wheelchair," 30th Annual International Conference of the IEEE Engineering in Medicine and Biology Society (submitted), 2008.

[11] R. C. Simpson and S. P. Levine, "Automatic adaptation in the NavChair Assistive Wheelchair Navigation System," Rehabilitation Engineering, IEEE Transactions on [see also IEEE Trans. on Neural Systems and Rehabilitation], vol. 7, pp. 452 - 4631999. 